\title{
MANAGEMENT OF CRISES DURING ANESTHESIA AND SURGERY. PART XV: SEPSIS
}

\author{
Salam N Asfar ${ }^{@}$ \& Jasim M Salman \\ ${ }^{\circledR} \mathrm{MB}, \mathrm{ChB}, \mathrm{MSc}$, Professor of Anesthesiology, College of Medicine, University of Basrah. ${ }^{\#} \mathrm{MB}, \mathrm{ChB}$, \\ DA, FICMS, Assist. Prof. \& Consultant Anesthesiologist, College of Medicine, University of Basrah, \\ Basrah, IRAQ.
}

\section{Sepsis}

Q epsis which is defined as systemic response to infection may potentially causes

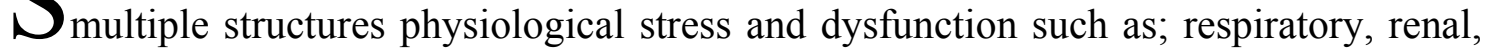
cardiovascular, and hematological. Because quick consideration should be paid to so many body systems, prepared approach during anesthesia for a septic patient is a must ${ }^{1}$. Patients with sepsis show signs of systemic inflammatory response syndrome, as result from generalized reaction to inflammation which may be caused by a wide range of infectious and non-infectious causes for example, burns, pancreatitis and multiple trauma ${ }^{2}$.

\section{Signs and laboratory results ${ }^{1,3}$ :}

Altered mental status

Tachypnea

Fever $\left(>38.3^{\circ} \mathrm{C}\right.$ ) or Hypothermia (core temperature $<36^{\circ} \mathrm{C}$ )

Heart rate $>90 / \mathrm{min}$

Arterial hypotension $(\mathrm{sBP}<90 \mathrm{mmHg})$

Arterial hypoxemia $(\mathrm{PaO} 2 / \mathrm{FiO} 2<300)$

Decreased capillary refill or mottling

Acute oliguria (urine output $<0.5 \mathrm{ml} / \mathrm{kg} / \mathrm{hr}$ for at least $2 \mathrm{hrs}$ despite adequate fluid resuscitation)

Ileus (absent bowel sounds)

Significant edema or positive fluid balance ( $>20 \mathrm{~mL} / \mathrm{kg}$ over 24 hours)

Hyperglycemia (plasma glucose $>140 \mathrm{mg} / \mathrm{dl}$ ) in the absence of diabetes

Leukocytosis (WBC count $>12,000 / \mu \mathrm{l}$ ), leukopenia (WBC count $<4,000 / \mu \mathrm{l}$ ) or normal WBC count with greater than $10 \%$ immature forms

High serum creatinine

High Plasma C-reactive

Coagulation abnormalities (INR $>1.5$ or a PTT $>60 \mathrm{sec}$ )

Thrombocytopenia (platelet count $<100,000 / \mu \mathrm{l}$ )

Hyperbilirubinemia (plasma total bilirubin $>4 \mathrm{mg} / \mathrm{dl}$ )

Unexplained metabolic acidosis

Evidence of disseminated intravascular coagulopathy

Postoperative respiratory failure

Failure to reverse 


\section{Management ${ }^{4}$}

\section{Emergency management}

Call for help

$100 \%$ oxygen

Bolus of crystalloid or colloid $10 \mathrm{ml} / \mathrm{kg} \mathrm{IV}$

Consider placing an arterial line

Consider an adrenaline infusion, titrate to achieve a mean blood pressure $>70 \mathrm{mmHg}$

\section{Further management}

Consider placing a central venous line, aim for a CVP of $>8 \mathrm{mmHg}$

Take samples for microscopy and culture of: blood, urine, Any other fluids

(e.g. bile, pus, ascites, CSF)

Record any prior antibiotics

Give appropriate antibiotics

Check hematology, coagulation status, biochemistry, blood gases

Place a urinary catheter

\section{Further care}

*Continue vigorous fluid resuscitation throughout

* Continue the adrenaline infusion

*If there is continuing instability send to the ICU. These instabilities may include:

-Cardiorespiratory compromise presenting as desaturation, hypovolaemia, and hypotension.

-Postoperative respiratory failure which may require re-intubation postoperatively.

-High risk patients are: ASA class III or above, diabetics, ICU patients, immunocompromised patients, instrumental urological procedures, instrumental enteric and biliary procedures, orthopaedic infections, urological sepsis, gynecological sepsis, biliary sepsis, pancreatitis, abscesses, and cellulitis or fasciitis ${ }^{2}$.

\section{References}

1. Matot I, Sprung CL. Definitions of sepsis. Intensive Care Med 2001;27(Sup):S3-9.

2. Muckart DJ, Bhagwanjee S. American College of Chest Physicians, Society of Critical Care Medicine Consensus Conference definitions of the systemic inflammatory response syndrome and allied disorders in relation to critically injured patients. Crit Care Med 1997;25:1789-1795.

3. Koichi Yuki and Naoka Murakami, Sepsis pathophysiology and anesthetic consideration. https://www.ncbi.nlm.nih.gov/pmc/articles/PMC4704087.

4. Myburgh JA, Chapman MJ, Szekely SM, Crisis management during anesthesia: sepsis, from qshc.bmj.com, 2010, Published by group.bmj.com. 